\title{
Open-pFind Enhances the Identification of Missing Proteins from Human Testis Tissue
}

Jinshuai Sun ${ }^{\ddagger} \S, \nabla$, Jiahui Shi ${ }^{\ddagger},, \nabla$, Yihao Wang ${ }^{\ddagger}, \nabla$, Shujia Wu", Liping Zhao ${ }^{\ddagger}$, , Yanchang $\mathrm{Li}^{\ddagger}$, Hong

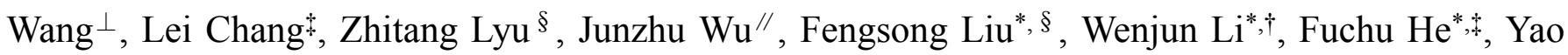
Zhang ${ }^{*}, \dagger, \hbar$, Ping $\mathrm{Xu}^{*}, \ldots, \delta, / /, \#$

${ }^{\dagger}$ State Key Laboratory of Biocontrol and Guangdong Provincial Key Laboratory of Plant Resources, School of Life Sciences, Sun Yat-Sen University, Guangzhou 510275, China

\$State Key Laboratory of Proteomics, Beijing Proteome Research Center, National Center for Protein Sciences (Beijing), Beijing Institute of Lifeomics, Beijing 102206, China

$\S$ Hebei Province Key Lab of Research and Application on Microbial Diversity, College of Life Sciences, Hebei University, Baoding, Hebei 071002, China

"Key Laboratory of Combinational Biosynthesis and Drug Discovery (Wuhan University), Ministry of Education, School of Pharmaceutical Science, Wuhan University, Wuhan 430072, China

${ }^{\perp}$ School of Public Health, North China University Science and Technology, Tangshan 063210, China

${ }^{\#}$ Guizhou University School of Medicine, Guiyang 550025, China

\section{*Corresponding Authors}

Ping Xu, E-mail: xuping @mail.ncpsb.org.

Yao Zhang, E-mail: zhangyaowsw@163.com.

Fuchu He, E-mail: hefc@bmi.ac.cn.

Wenjun Li, E-mail: liwenjun3@mail.sysu.edu.cn.

Fengsong Liu, E-mail: liufengsong@hbu.edu.cn.

\section{Supporting information files: Figure S1-S7\&Table S1-S6}




\section{Supplemental Methods}

\section{The Calculation of FDRs from Open-pFind and pFind Searching Datasets}

Open-pFind adopts two searching modes, open searching and restricted searching. After processing the open and restricted searching, the results are merged and reranked again based on the q-value. Finally, PSMs, peptides and proteins are individually filtered in line with the specified thresholds (1\% FDR at each level).

In this study, we filtered the results of PSMs and peptides based on protein information, leaving only PSMs and peptides with protein reference (1\% FDR).

Simply superimposing the datasets from the multi-protease method will result in a higher FDR due to the random distribution of false positives and the much smaller overlaps than true positives. After processing three different datasets separately, we used q-value to combine these results. For the same protein identification, the smallest q-value was kept from different datasets. Based on these integrated qvalues, the combined results were reranked. Similarly, the cutoff score of q-value was accepted when $1 \%$ of decoy/target had accumulated.

\section{The Calculation of FDRs from MaxQuant Searching Datasets}

MaxQuant calculates the FDR by using the posterior error probability (PEP). To determine the cutoff score for $1 \%$ FDR at peptide level, it sorts all identified peptides from the forward and the reverse database by their PEP, starting with the best. Peptides are accepted until 1\% of reverse hits/forward hits had accumulated.

After assembling peptide hits into protein hits, the software assigns to each protein group a PEP by multiplying their peptide PEPs. Similarly, it uses the corresponding PEP score to control the 1\% FDR at protein level.

To combine datasets from the multi-protease method, three sets of group parameters were set in one task to cope with different digestions.

\section{The Calculation of FDRs from Proteome Discoverer Searching Datasets}

Proteome Discoverer 2.2 assigns q-values and PEPs to a set of target and decoy PSMs generated by Sequest HT according to the quality of the match. Proteome Discoverer 2.2 calculates the FDR by using the rank 1 target PSM and the rank 1decoy PSM for each spectrum. It sorts all target and decoy PSMs by score and determines the score threshold by successively decreasing the score threshold value until the quotient of decoy and target PSMs reaches the specified target FDR (1\%).

As for peptide FDR, Proteome Discoverer 2.2 calculates q-value and PEPs for peptides engaging the quality algorithm at first. Then peptide confidences are assigned based on the target FDRs (Strict: 1\%, Relaxed: 5\%) for peptides.

As for protein FDR, Proteome Discoverer 2.2 calculates a score based on the PEP values of the PSMs and uses it to rank the list of proteins, then adjusts the value threshold to make protein FDR less than $1 \%$.

In this study, our datasets obtained from different proteases were recreated by multi-consensus processing in the Proteome Discoverer 2.2.

Acknowledge that not all proteins surviving the threshold are "confidently identified". 


\section{Name}

Figure S-1

Figure S-2

Figure S-3

Figure S-4

Figure S-5

Figure S-6

Figure S-7

\section{Supplementary Figure/Table Legends}

Venn diagram of the identified proteins by four search engines from three different data sets. (A-C) Comparison of the identified proteins by four search engines from testis samples digested with Glu-C (A), Lys-C (B), and Trypsin (C), respectively.

Open-pFind is superior to three other search engines in the consistently identified proteins from four search engines based on three large-scale data sets. (A-B) PSMs and sequence coverage evaluation of the consistently identified proteins from Glu-C digesting datasets, respectively. (C-D) PSMs and sequence coverage evaluation of the consistently identified proteins from Lys-C digesting datasets, respectively.

Open-pFind shows an advantage greater than the three other search engines in the separately identified proteins based on three large-scale data sets. (A) Protein length, (B) The unique peptides, (C) Sequence coverage evaluation of the proteins separately identified by four search engines from three digested datasets.

Venn diagram of the MP candidates identified by four search engines from three different data sets. (A) Comparison of the identified MP candidates by four search engines in Trysin, (B) Lys-C, (C) Glu-C digesting datasets.

Performance evaluation of the MP candidates identified by Open- pFind with three data sets. (A) Comparison of the identified MP candidates by Open-pFind. (B) The chromosome distribution of the total identified MP candidates by Open-pFind.

Verification of the unique peptides for the five confident MPs with the synthesized peptide.

Verification of the unique peptides for the three MP candidates with the synthesized peptide

Table S-1 Criteria and results of testis proteomics data searched with four engines.

Table S-2 Identified gene products with four search engines on testis proteomics datasets.

Table S-3 Identified unique proteins with four search engines on testis proteomics datasets.

Table S-4 Identification of 5 verified and 3 MP candidates in this study.

Table S-5 Information of the other obtained MP candidates identified after SAAVs selecting in this study.

Table S-6 The identified peptides of the 124 MP candidates in this study. 
A

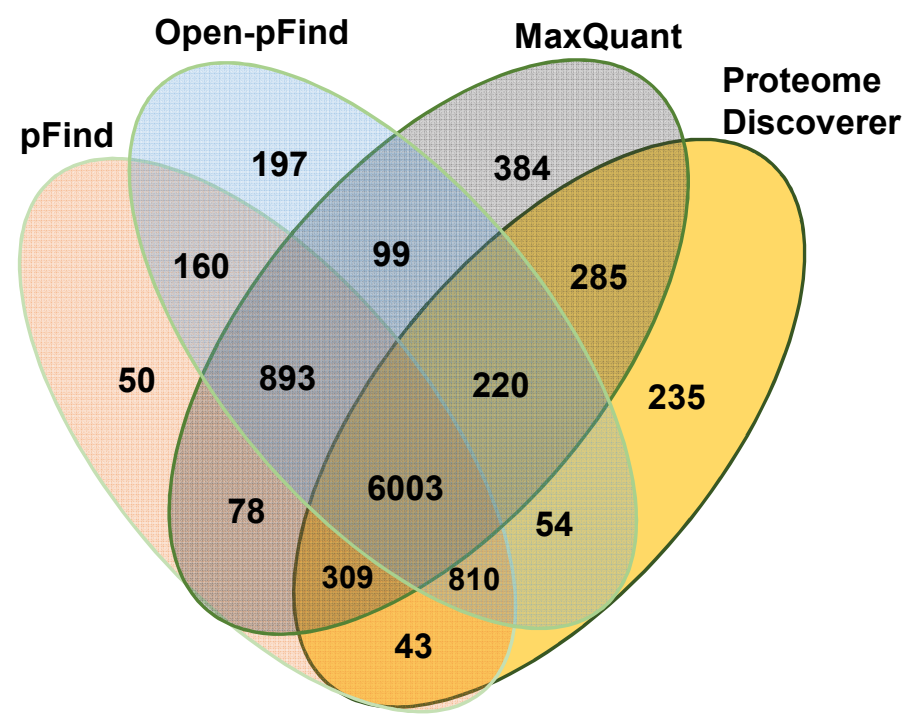

B

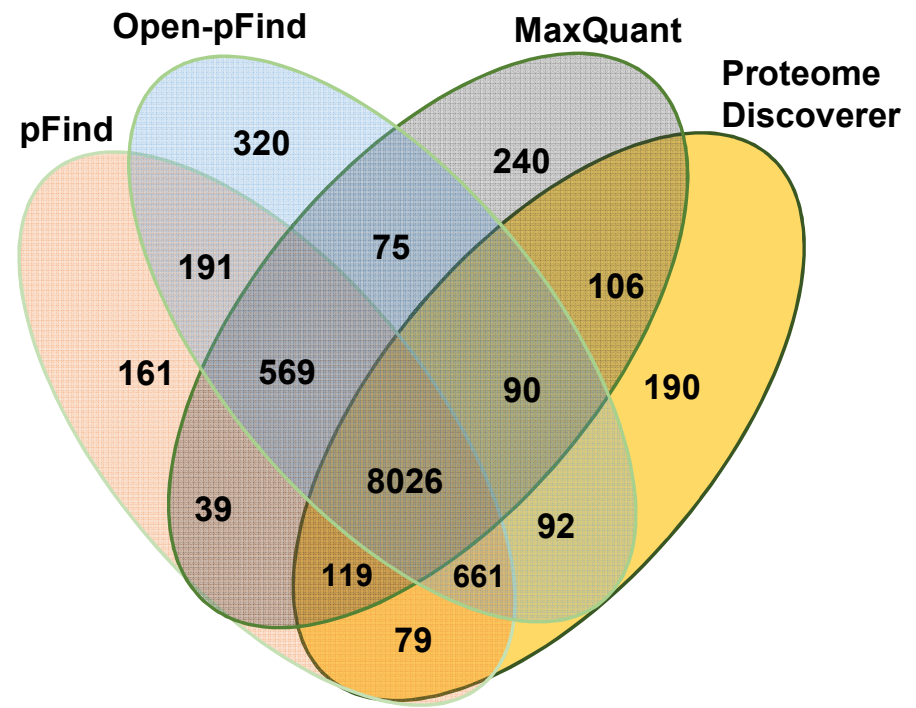

Lys-C

C

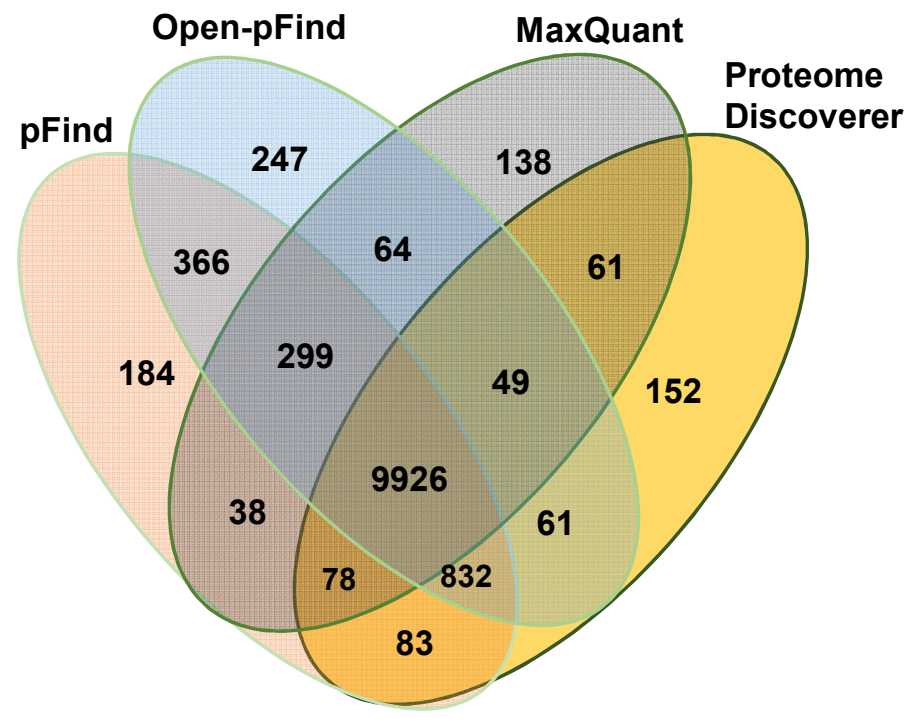

\section{Trypsin}

Figure S-1. Venn diagram of the identified proteins by four search engines from three different data sets. (A-C) Comparison of the identified proteins by four search engines from testis samples digested with Glu-C (A), Lys-C (B), and Trypsin (C), respectively. 


\section{Glu-C}
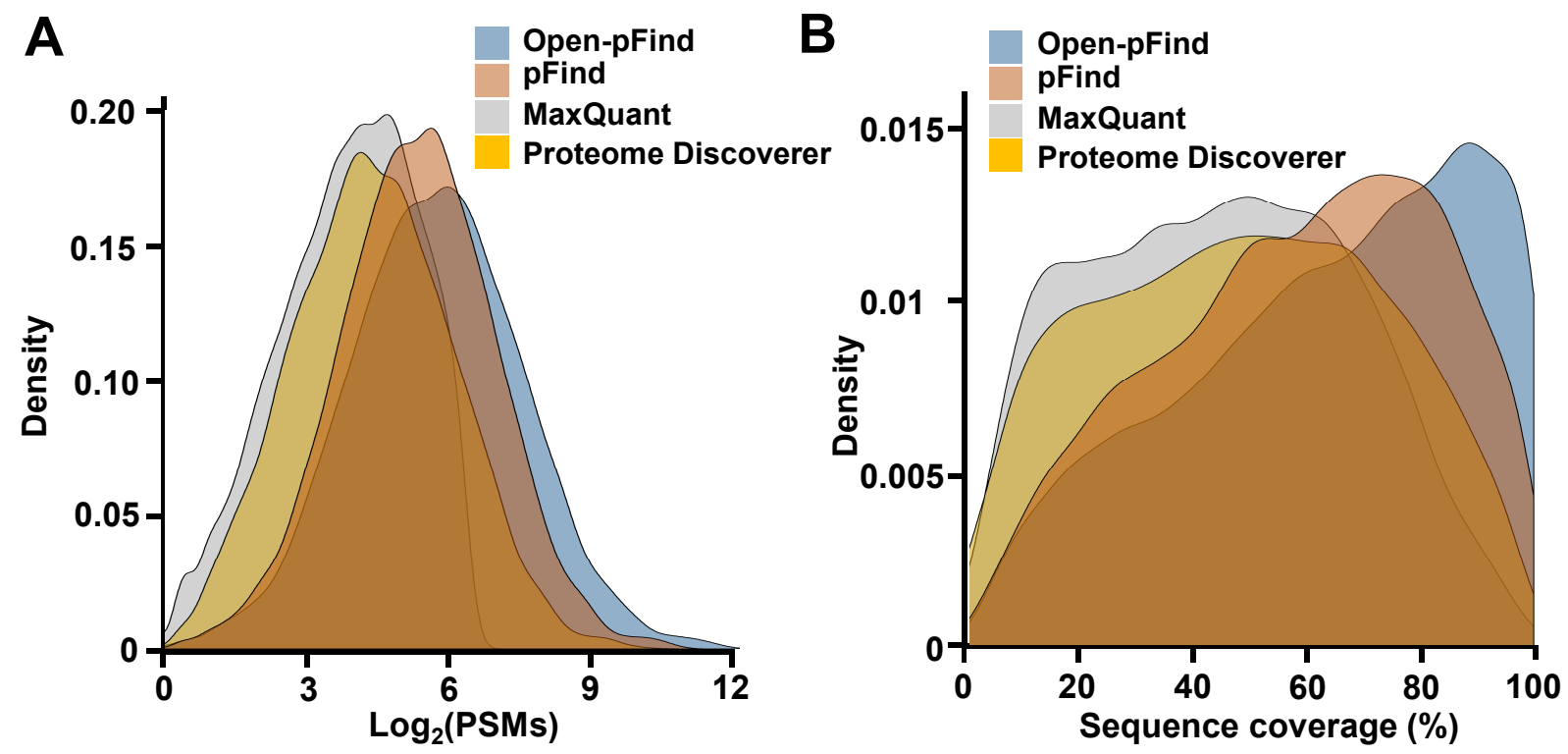

\section{Lys-C}
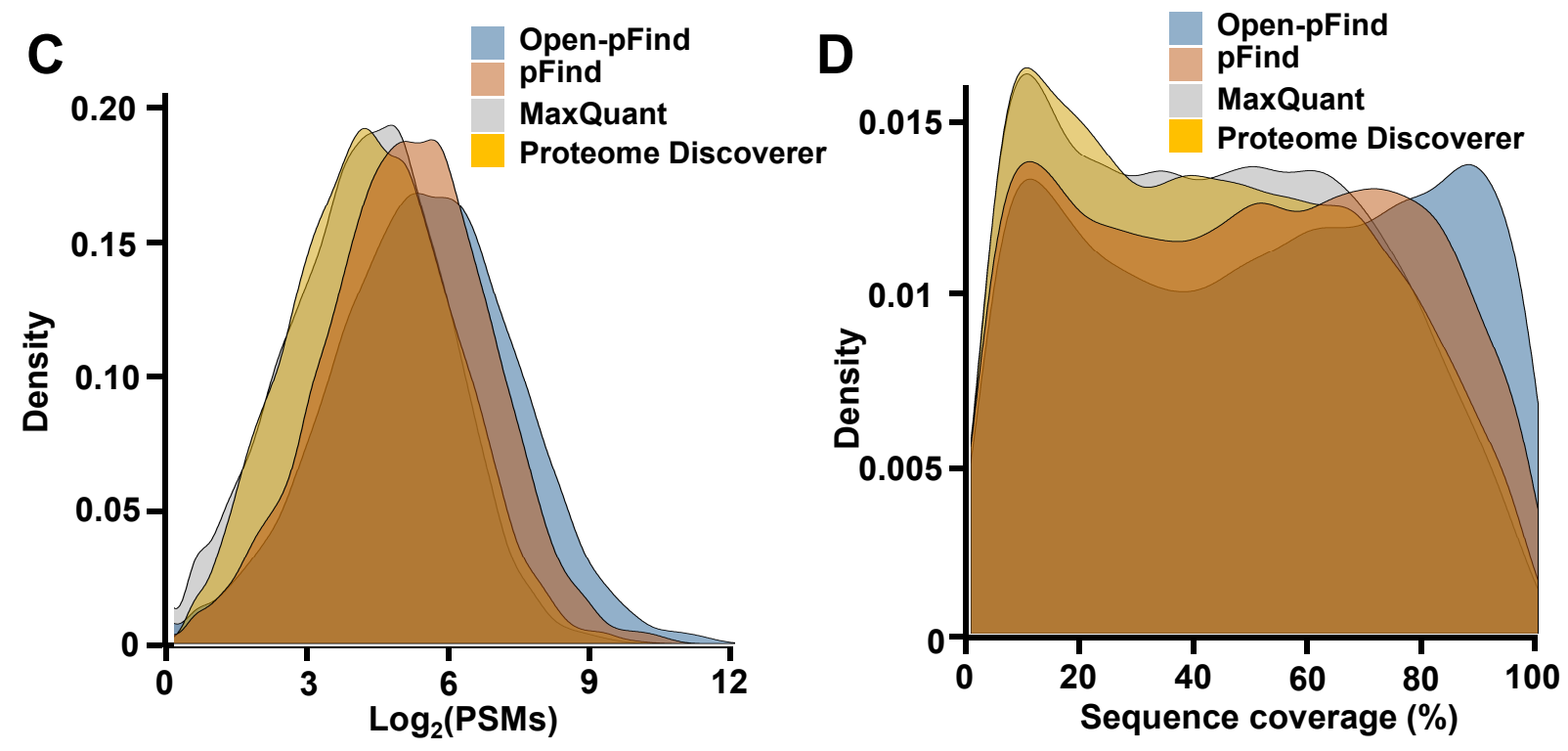

Figure S-2. Open-pFind is superior to three other search engines in the consistently identified proteins from four search engines based on three large-scale data sets. (A-B) PSMs and sequence coverage evaluation of the consistently identified proteins from Glu-C digesting datasets, respectively. (C-D) PSMs and sequence coverage evaluation of the consistently identified proteins from Lys-C digesting datasets, respectively. 
A $\quad$ Open-pFind $\square$ pFind $\square$ MaxQuant $\square$ Proteome Discoverer

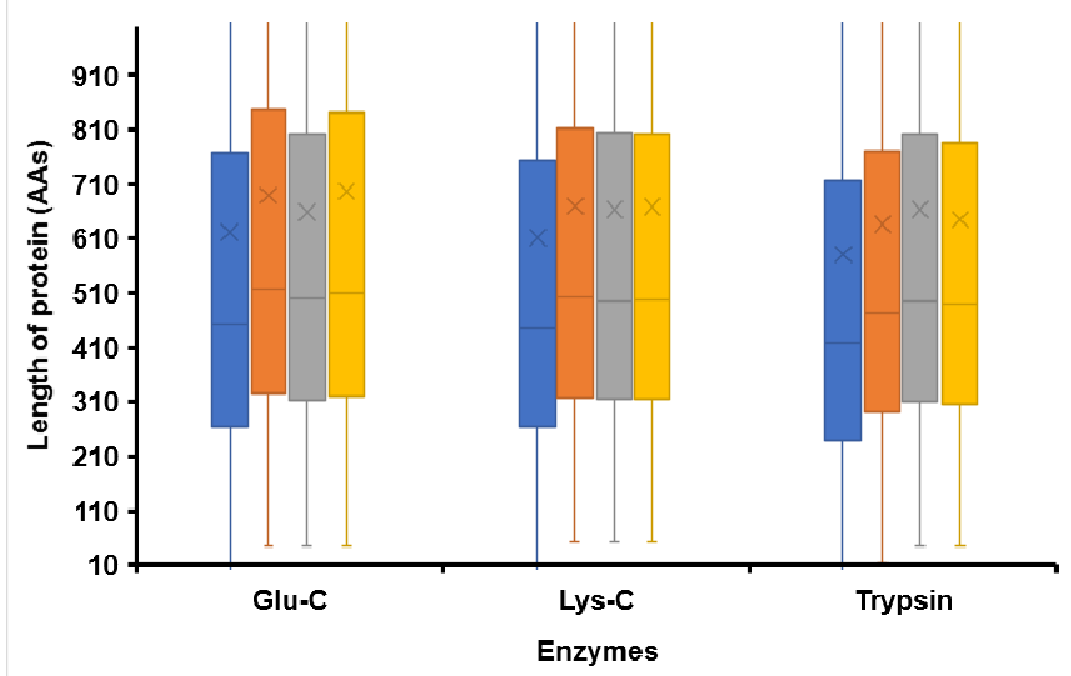

B $\square$ Open-pFind $\square$ pFind $\square$ MaxQuant $\square$ Proteome Discoverer

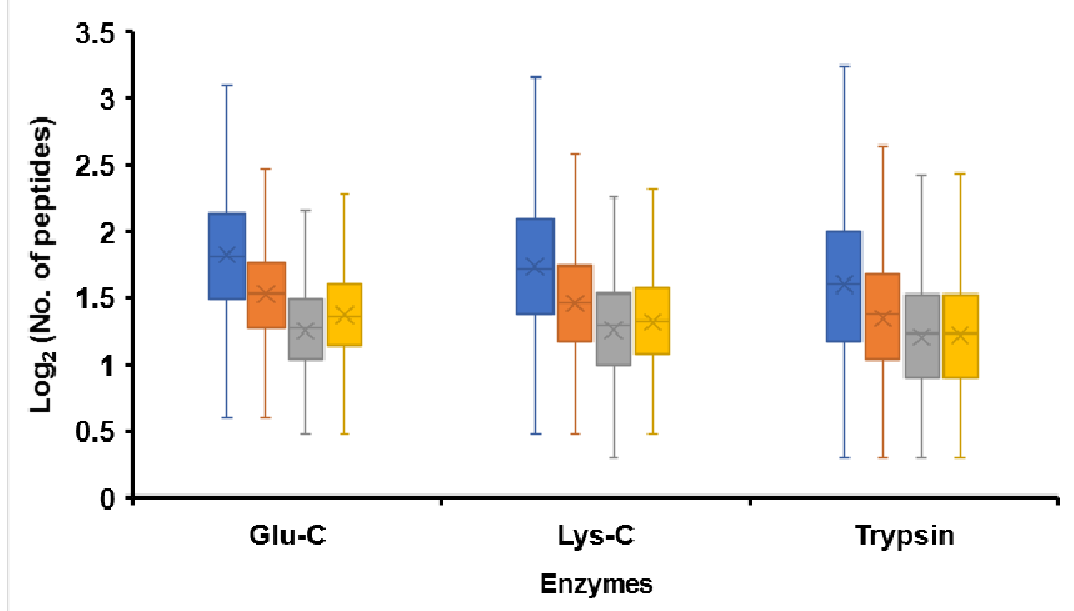

\section{C $\square$ Open-pFind $\square$ pFind $\square$ MaxQuant $\square$ Proteome Discoverer}

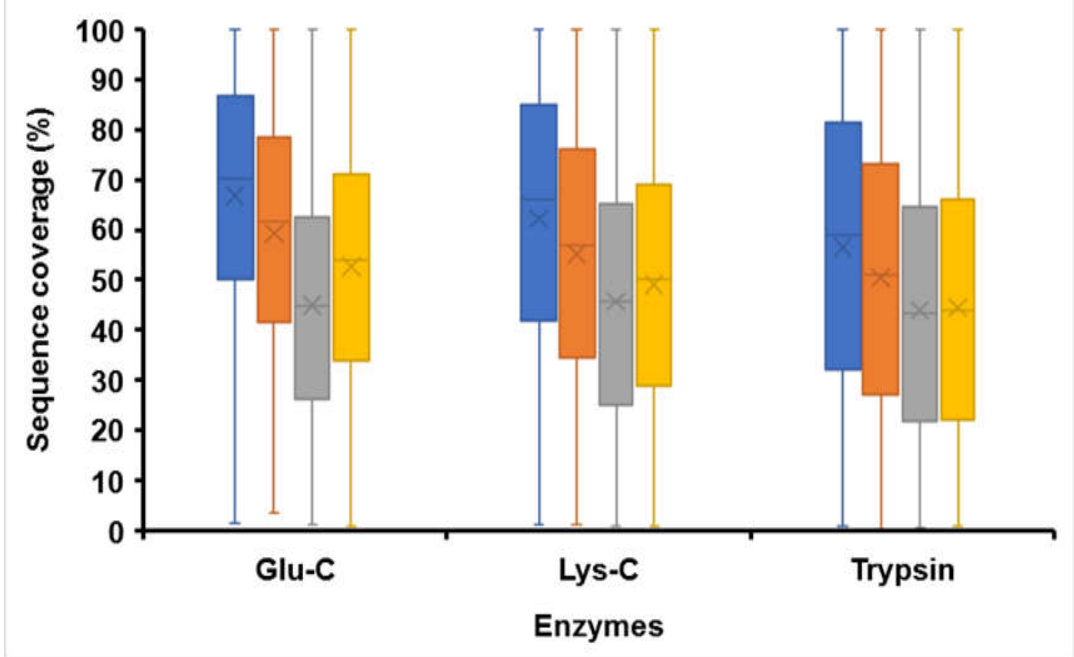

Figure S-3. Open-pFind shows an advantage greater than the three other search engines in the separately identified proteins based on three large-scale data sets. (A) Protein length, (B) The unique peptides, (C) Sequence coverage evaluation of the proteins separately identified by four search engines from three digested datasets. 
A

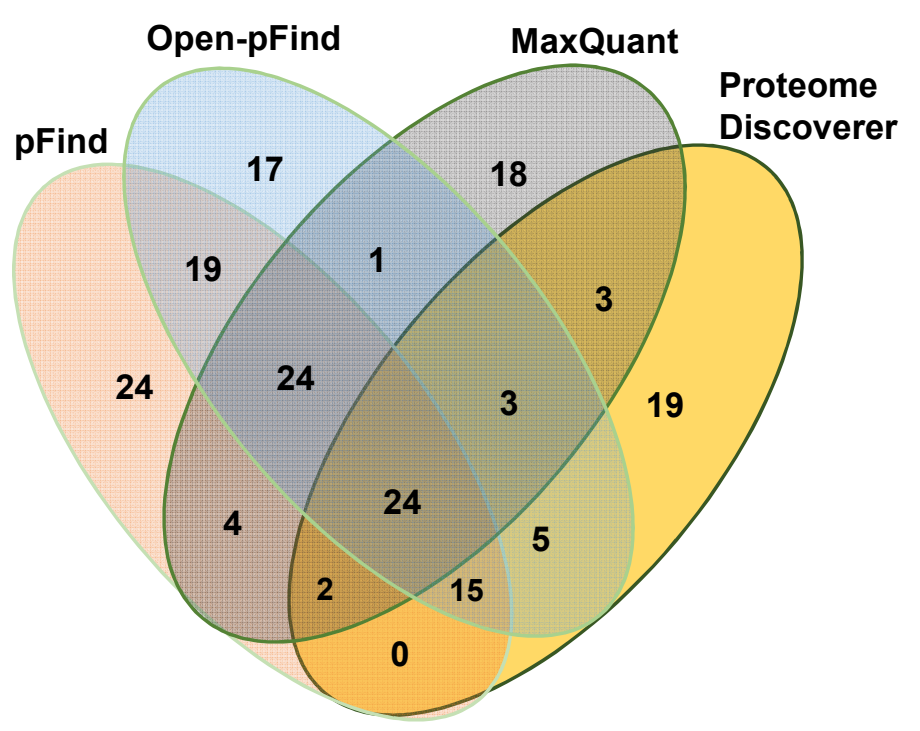

\section{Trypsin}

B

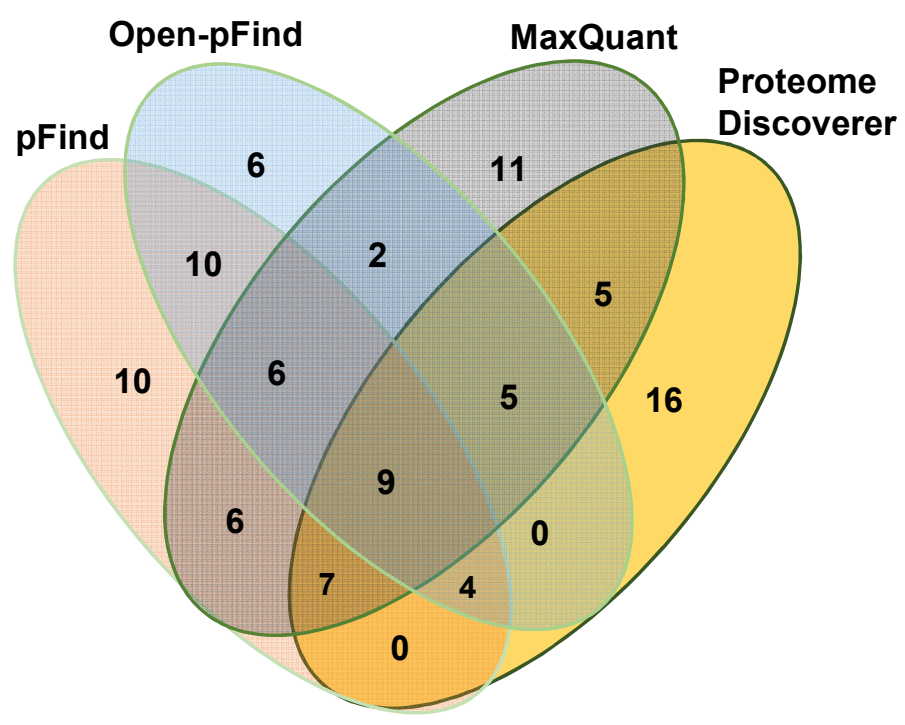

\section{Lys-C}

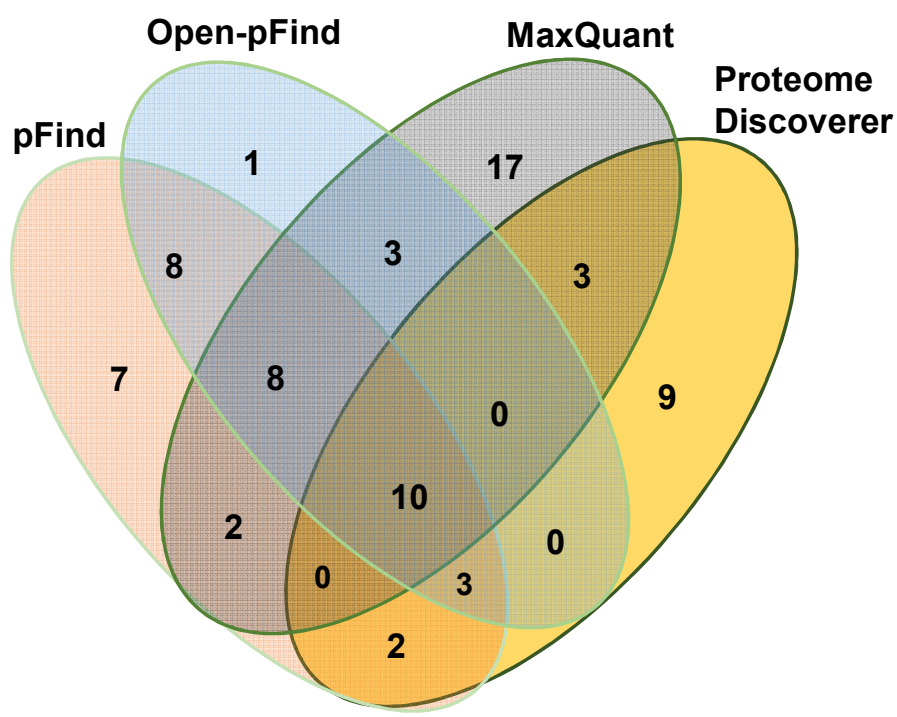

Glu-C

Figure S-4. Venn diagram of the MP candidates identified by four search engines from three different data sets. (A) Comparison of the identified MP candidates by four search engines in Trysin, (B) Lys-C, (C) Glu-C digesting datasets. 
A

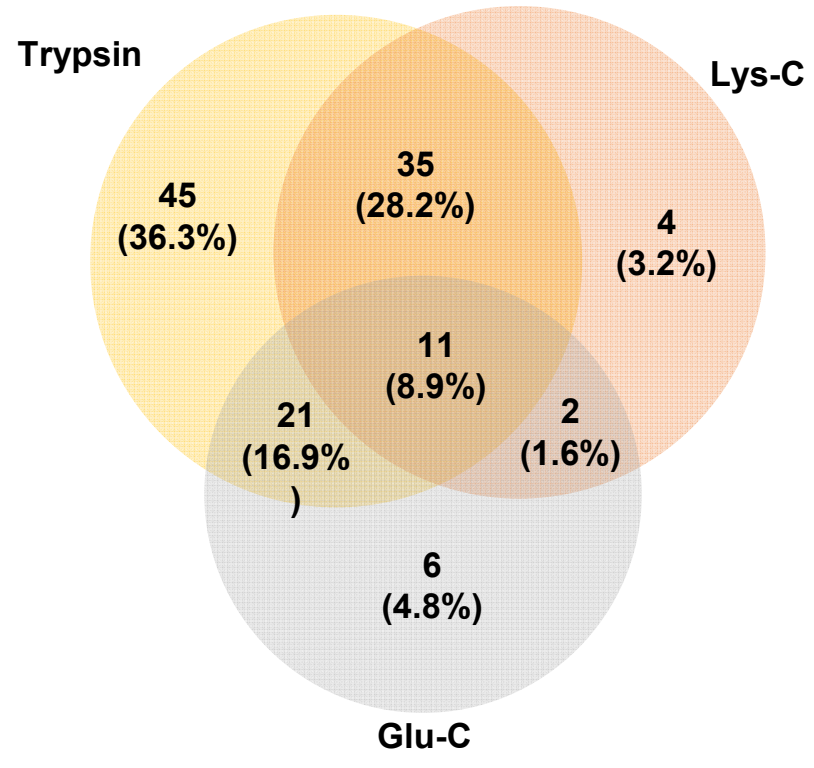

B

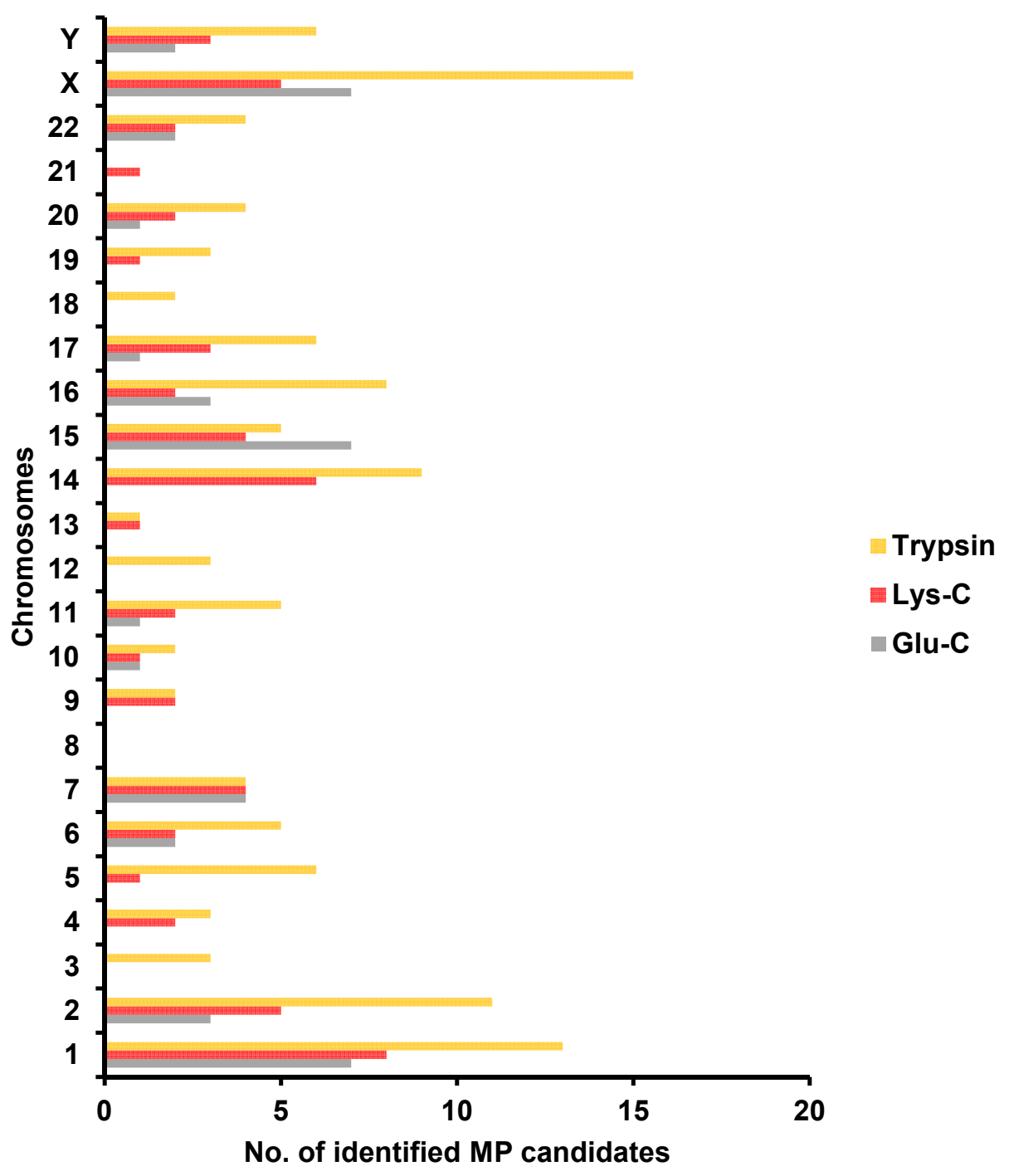

Figure S-5. Performance evaluation of the MP candidates identified by Open- $p$ Find with three data sets. (A) Comparison of the identified MP candidates by Open-pFind. (B) The chromosome distribution of the total identified MP candidates by Open-pFind. 


\section{Q8TAA1}

A

8.14E5 $2+$

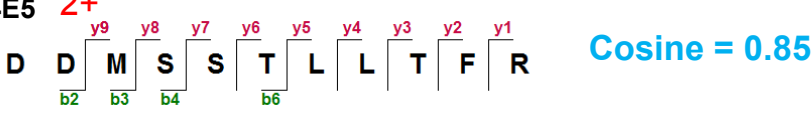

Original

Precursor $\mathrm{m} / \mathrm{z}$ : 643.310

Mass error: $2.850 \mathrm{ppm}$

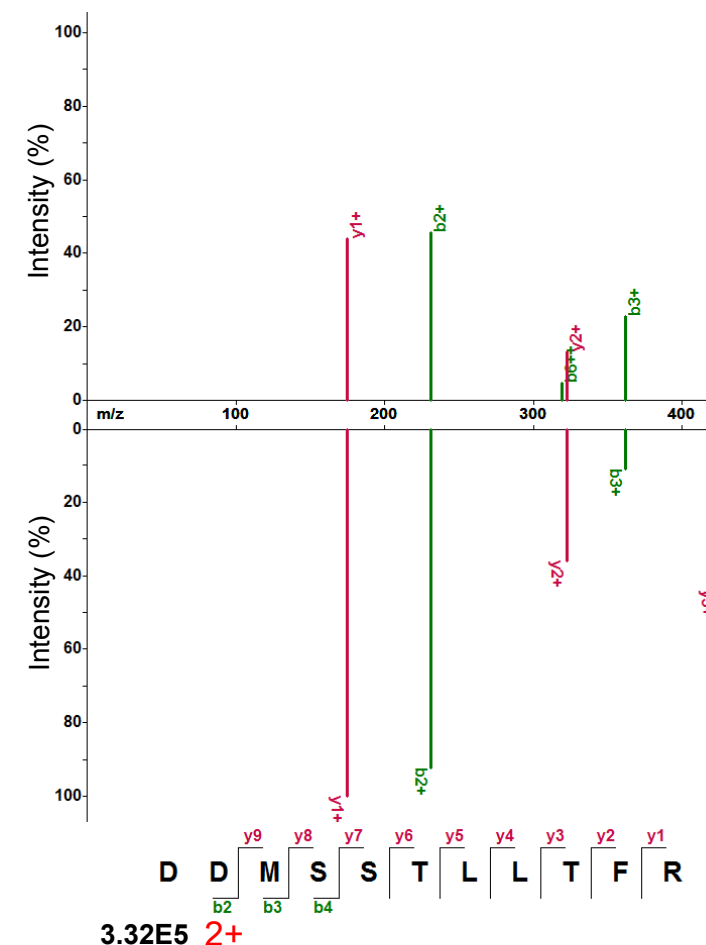

Synthetic

Precursor $\mathrm{m} / \mathrm{z}: 643.308$

Mass error: 0.296 ppm

3.32E5 $2+$

B

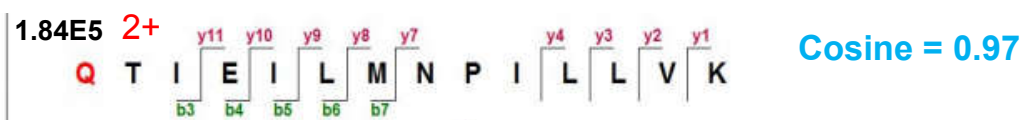

Original

$100-$ Gln->pyro-Glu[AnyN-termQ]

Precursor m/z: 804.481

$\overbrace{}^{\circ}$

(

Mass error: 7.554 ppm

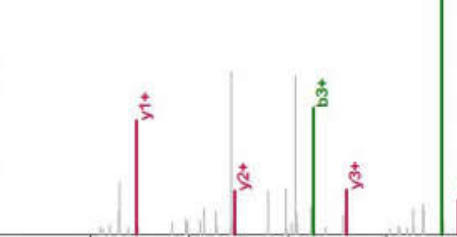

$\left.\left.\right|^{+1} \quad\right|^{+}$
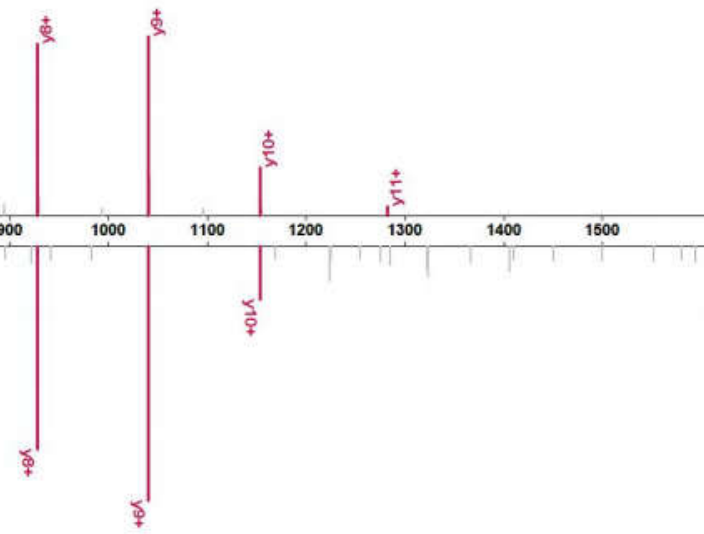

索

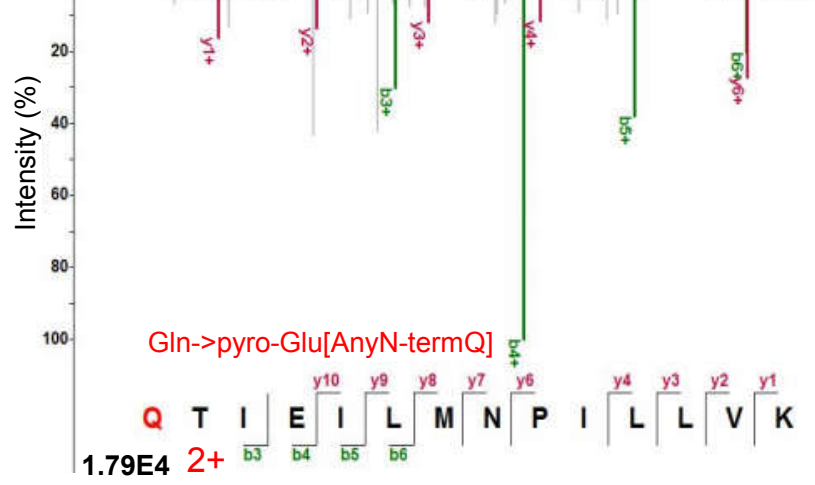

Synthetic

Precursor m/z: 804.475

Mass error: $\mathbf{- 0 . 7 5 8 ~ p p m ~}$

Figure S-6. Verification of the unique peptides for the five confident MPs with the synthesized peptide. 


\section{Q3ZLR7}

A

6.47E5 $3+$

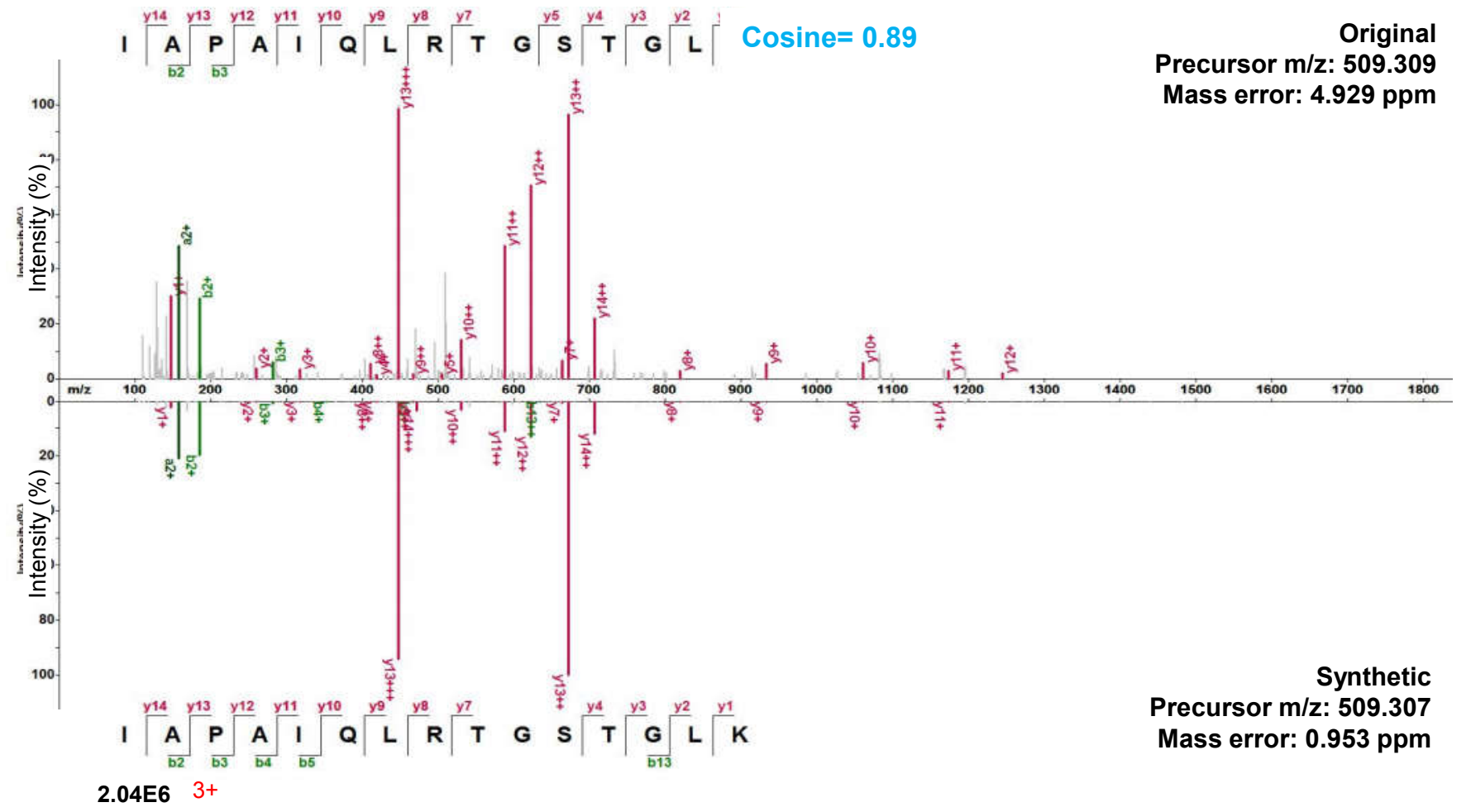

$B^{8.00 E 5} 3+$

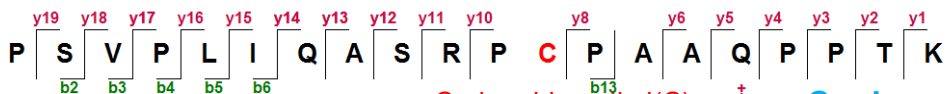

Original

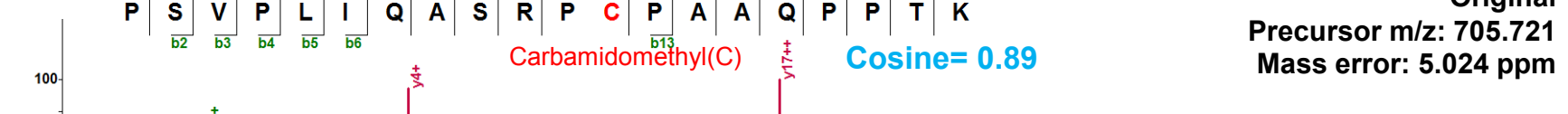
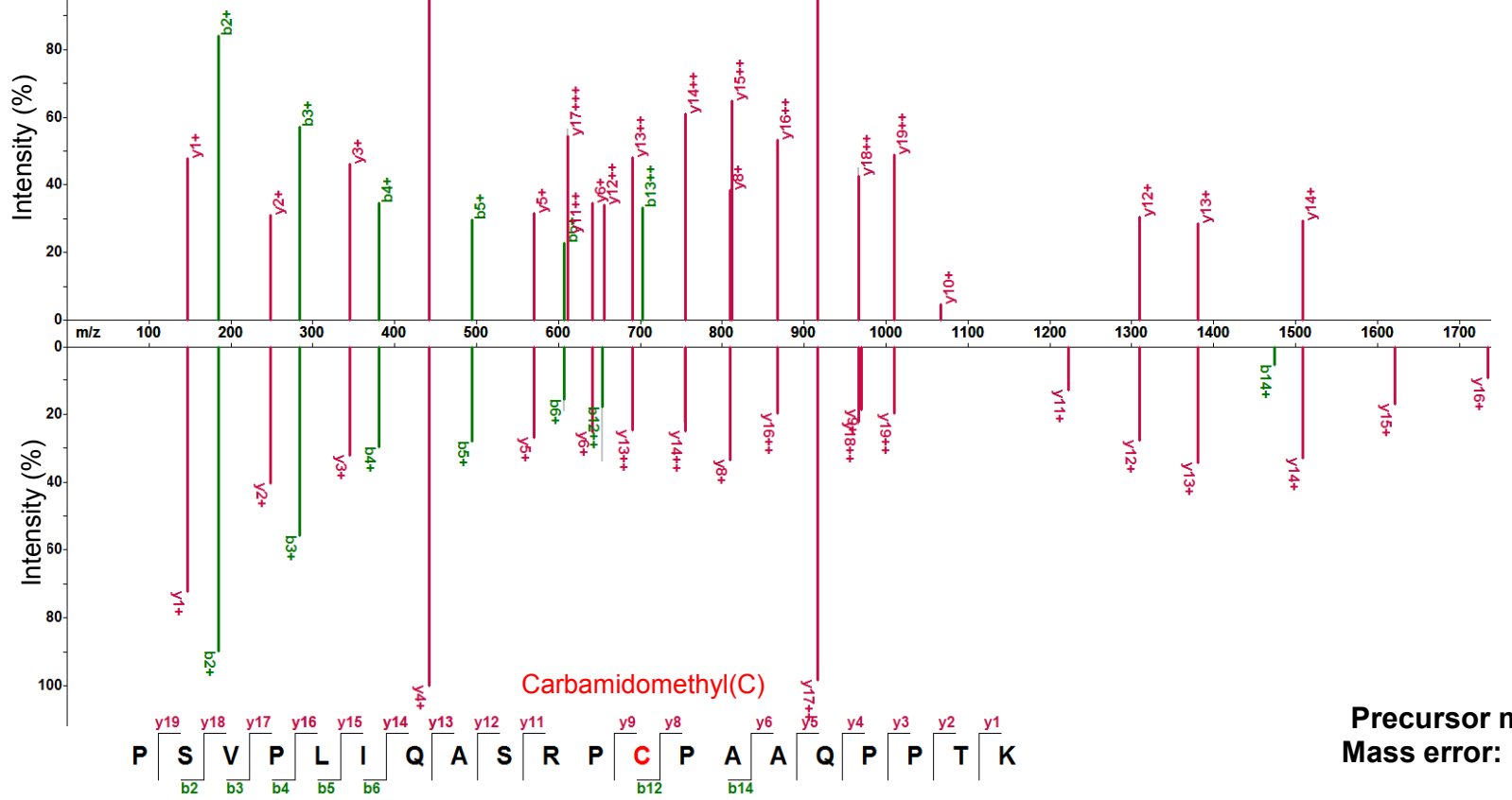

Synthetic

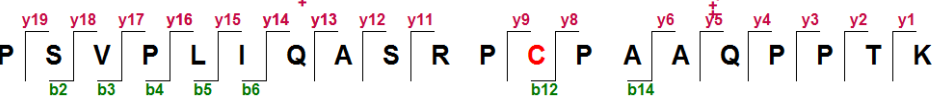

Precursor m/z:705.717

1.06E7 3+

Mass error: $-0.697 \mathrm{ppm}$ 


\section{A0A1B0GVM6}

A 6.09E5 5+

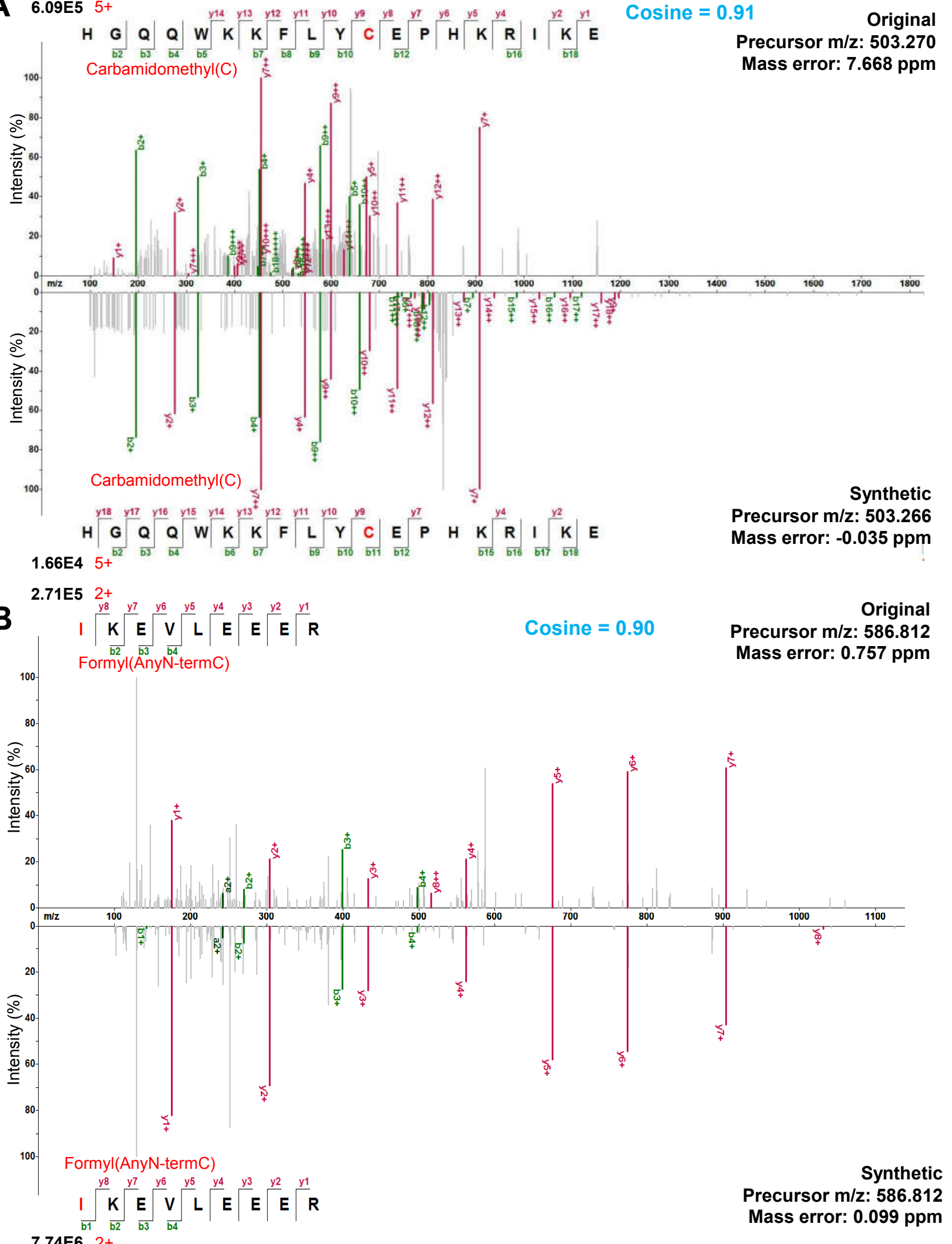




\section{Q5T1N1}

A

2.46E5 2+

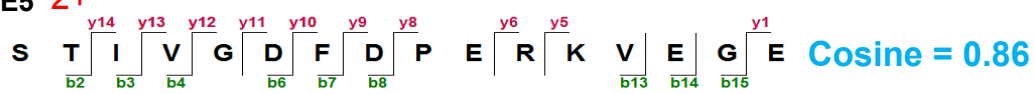

Original

Precursor m/z: 889.437
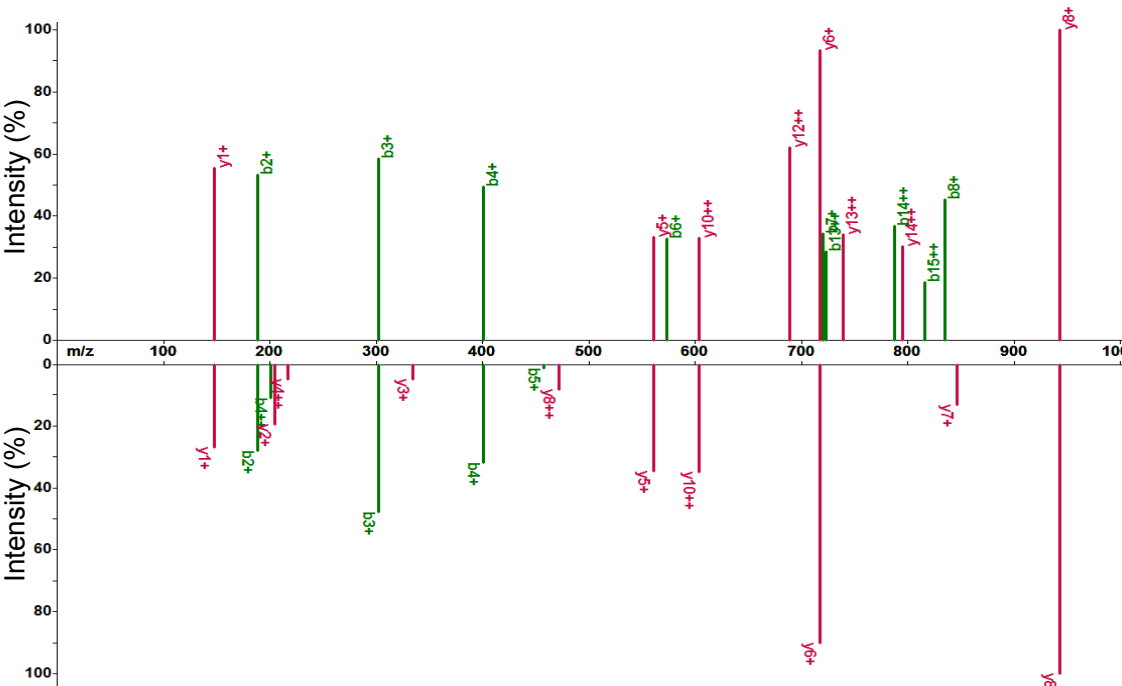

Mass error: $3.589 \mathrm{ppm}$

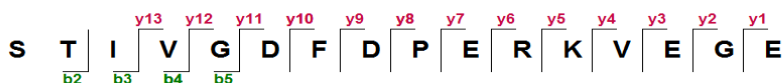

Synthetic

Precursor m/z: 889.434 Mass error: $-0.0 .19 \mathrm{ppm}$ 8.25E6 2+

B 1.29E6 3+

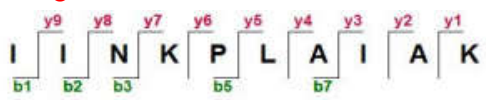

Cosine $=0.87$

Original

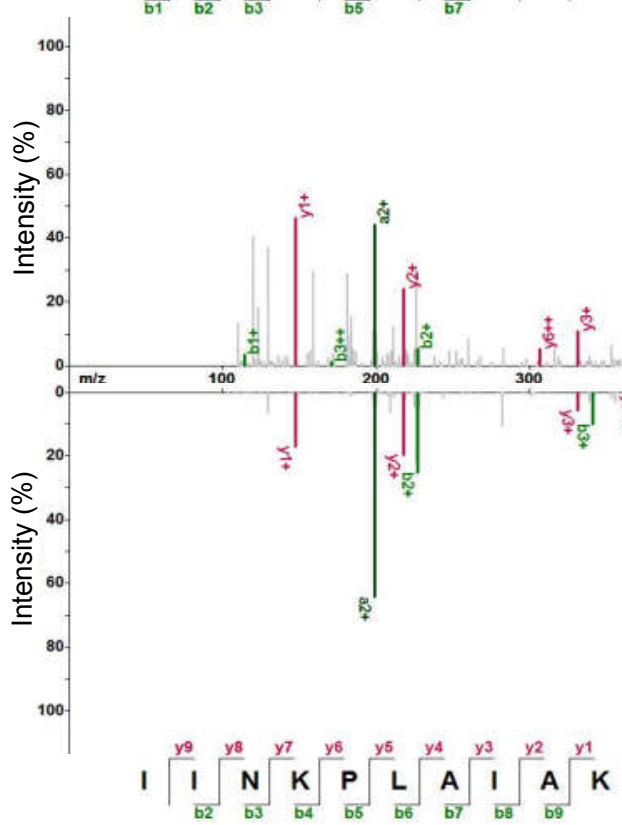

Precursor m/z: 360.911

Mass error: 5.252 ppm

$6.91 \mathrm{E} 63+$

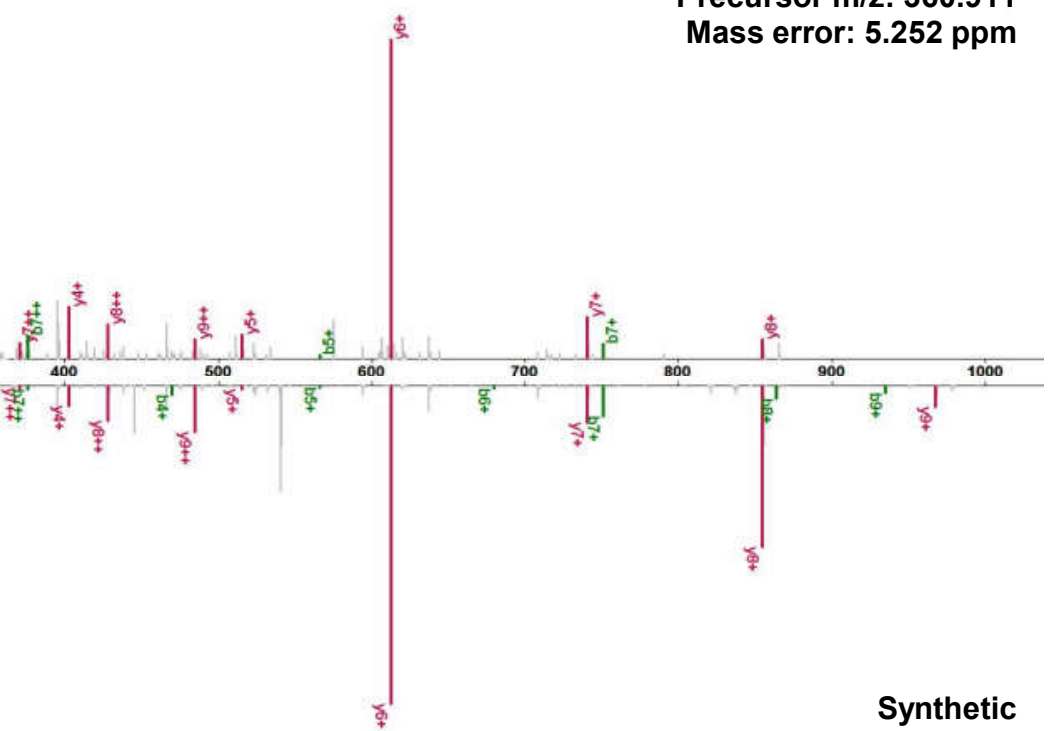

Precursor m/z: 360.909

Mass error: -0.266 ppm 


\section{C}

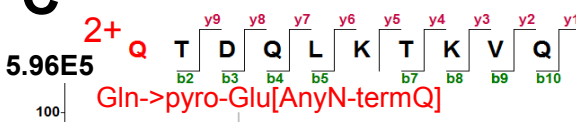

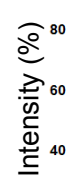

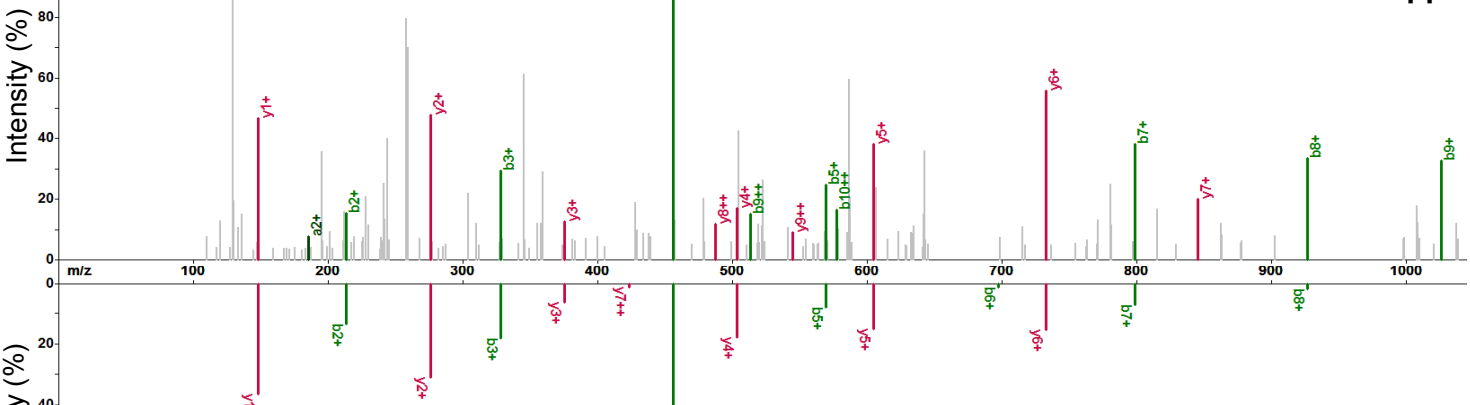

$\frac{\mathbb{T}^{4}}{4}$

$\stackrel{\Phi}{\stackrel{ \pm}{\subseteq}} 60$

80 


\section{Q8WW27}

A 7.27E5 2+

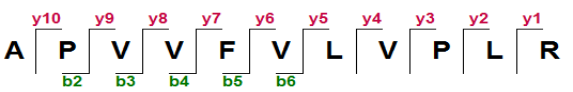

Original

Precursor m/z: 605.392

Cosine $=0.85$

Mass error: $4.327 \mathrm{ppm}$

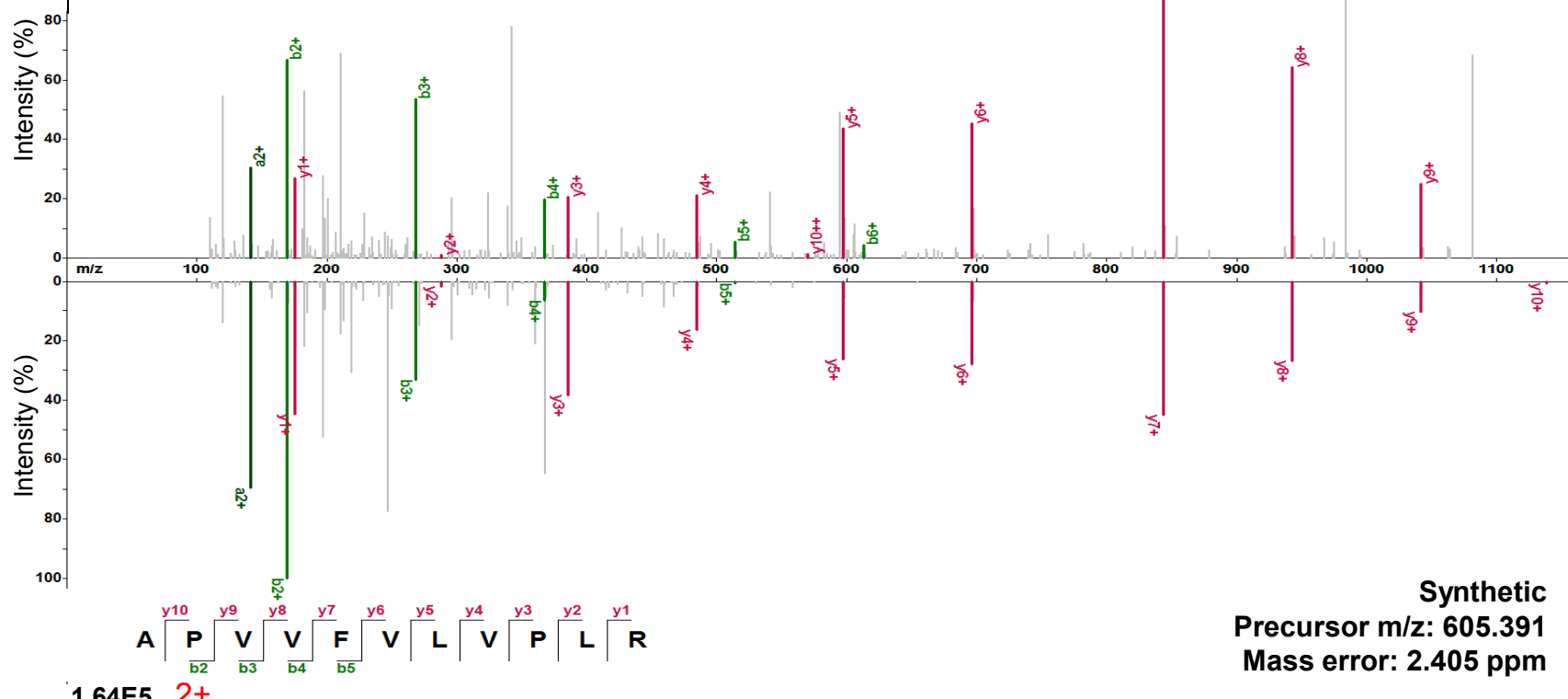

B

3.23E4 2+

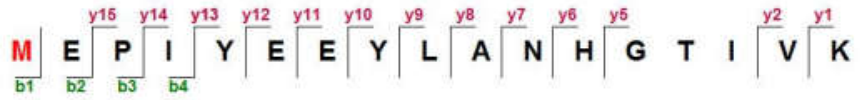

Acetyl(ProteinN-term)

Cosine $=0.94$

Original

Precursor m/z: 1025.001

Mass error: $6.046 \mathrm{ppm}$

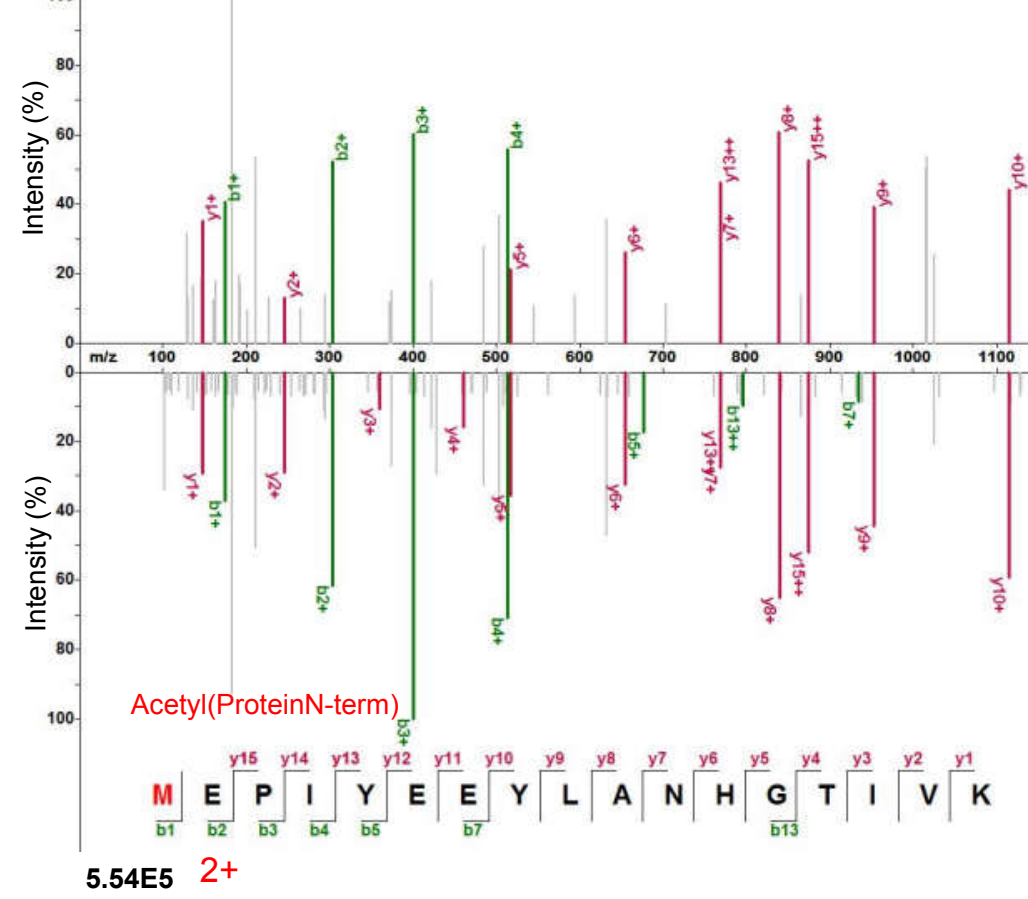

Synthetic

Precursor m/z: 1025.004

Mass error: $0.360 \mathrm{ppm}$ 


\section{Q8TF08}

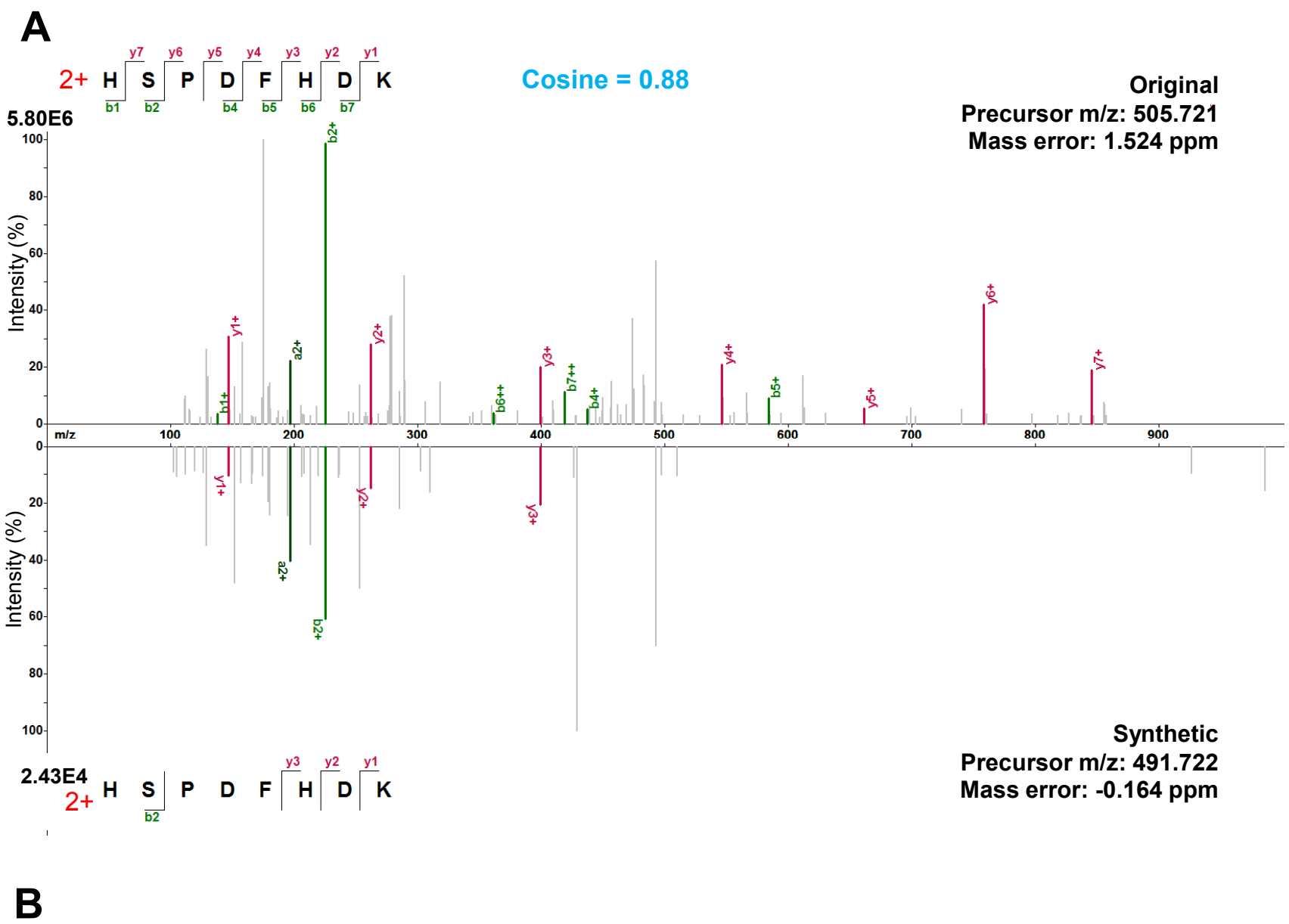

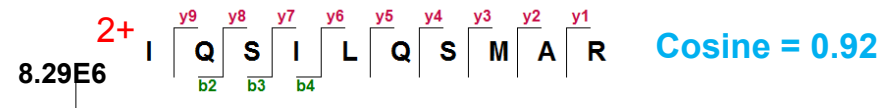

Original

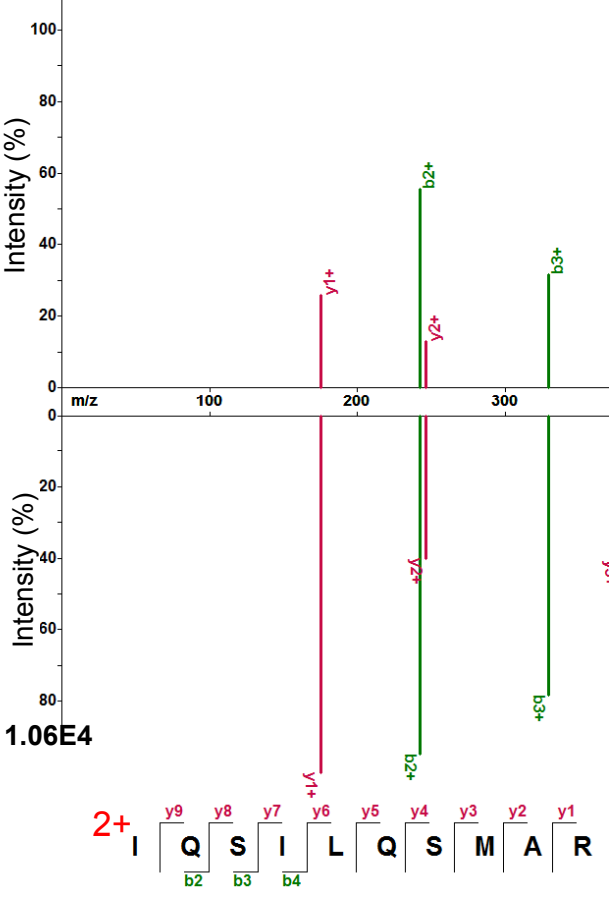

Precursor m/z: 573.820

Mass error: $2.311 \mathrm{ppm}$

Figure S-7. Verification of the unique peptides for the three MP candidates with the synthesized peptide 


\section{Q5T5N4}

A 5.85E5 2+

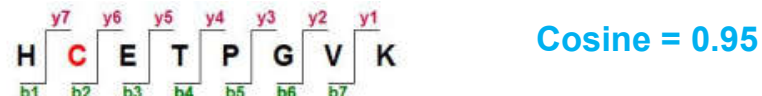

100. Carbamidomethyl(C) $\stackrel{+}{5}$

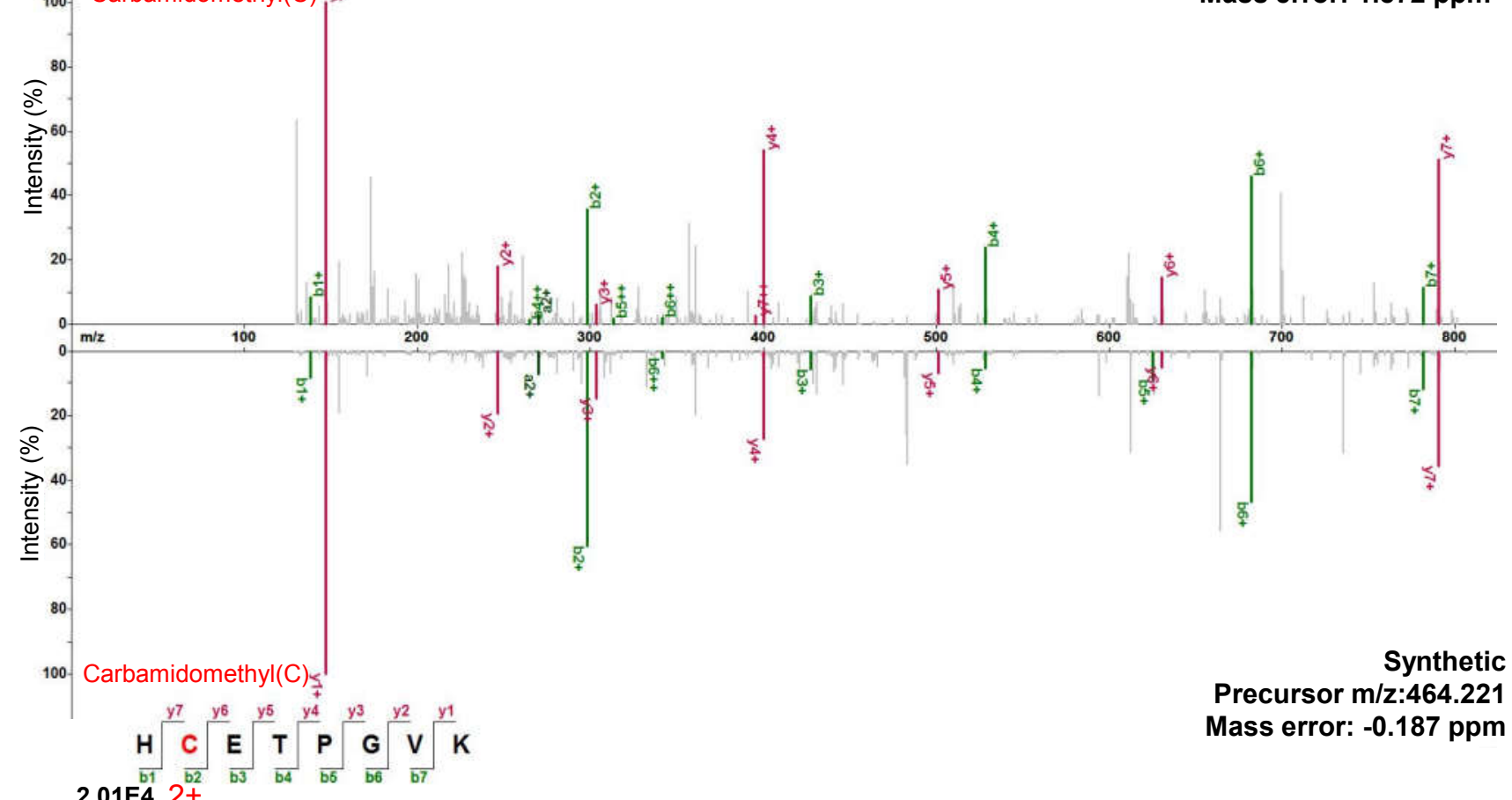

B

7.69E5 2+

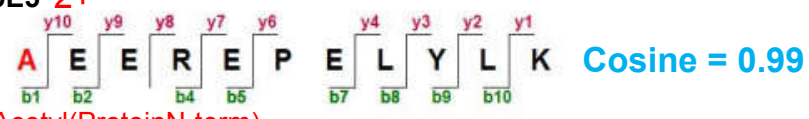

Original

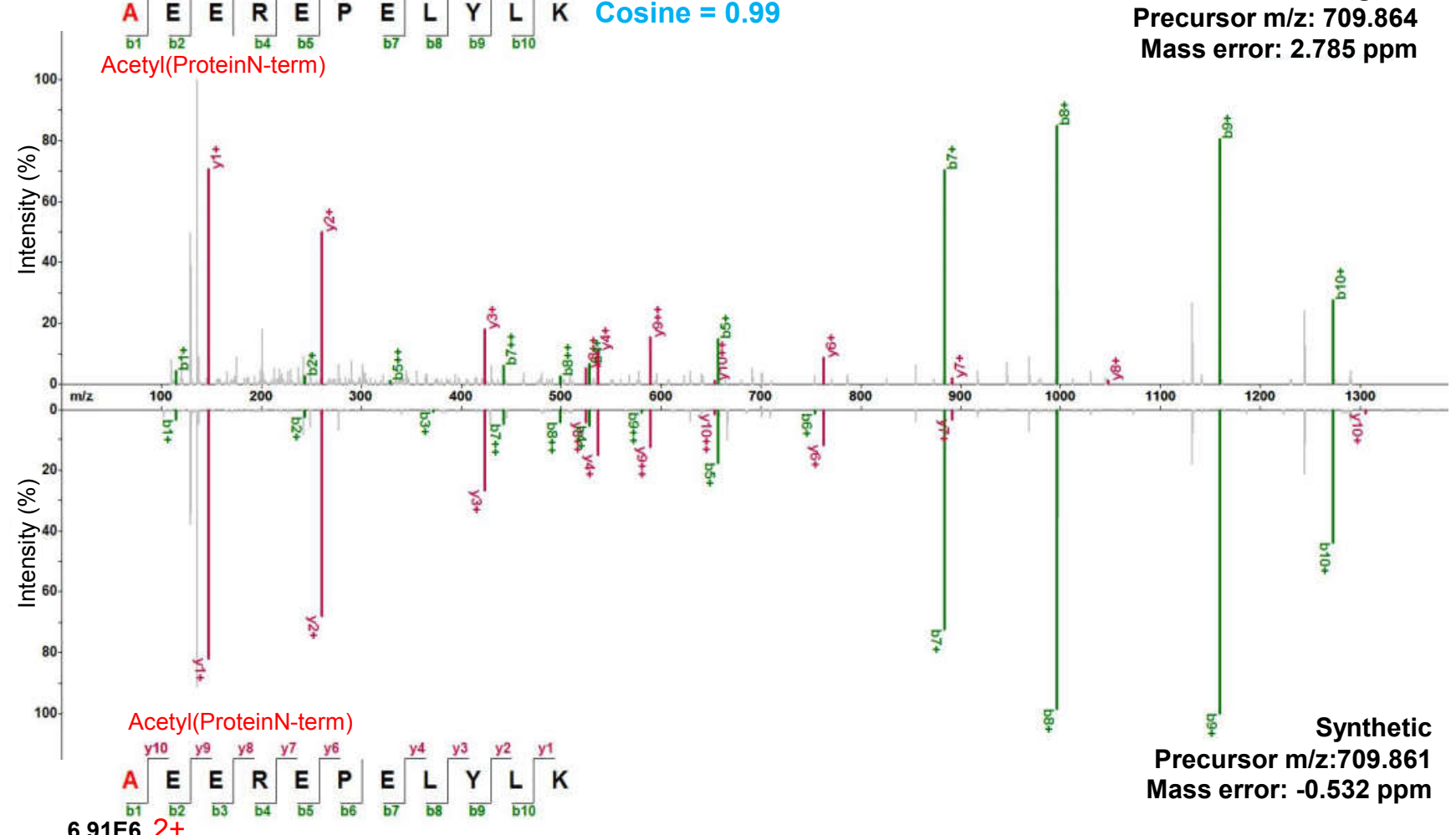




\section{A8MXV6}

A 1.09E6 3+

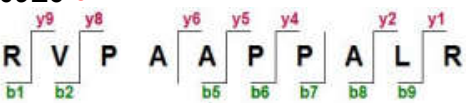

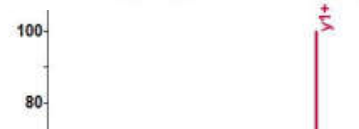

.

Mass error: 4.194 ppm

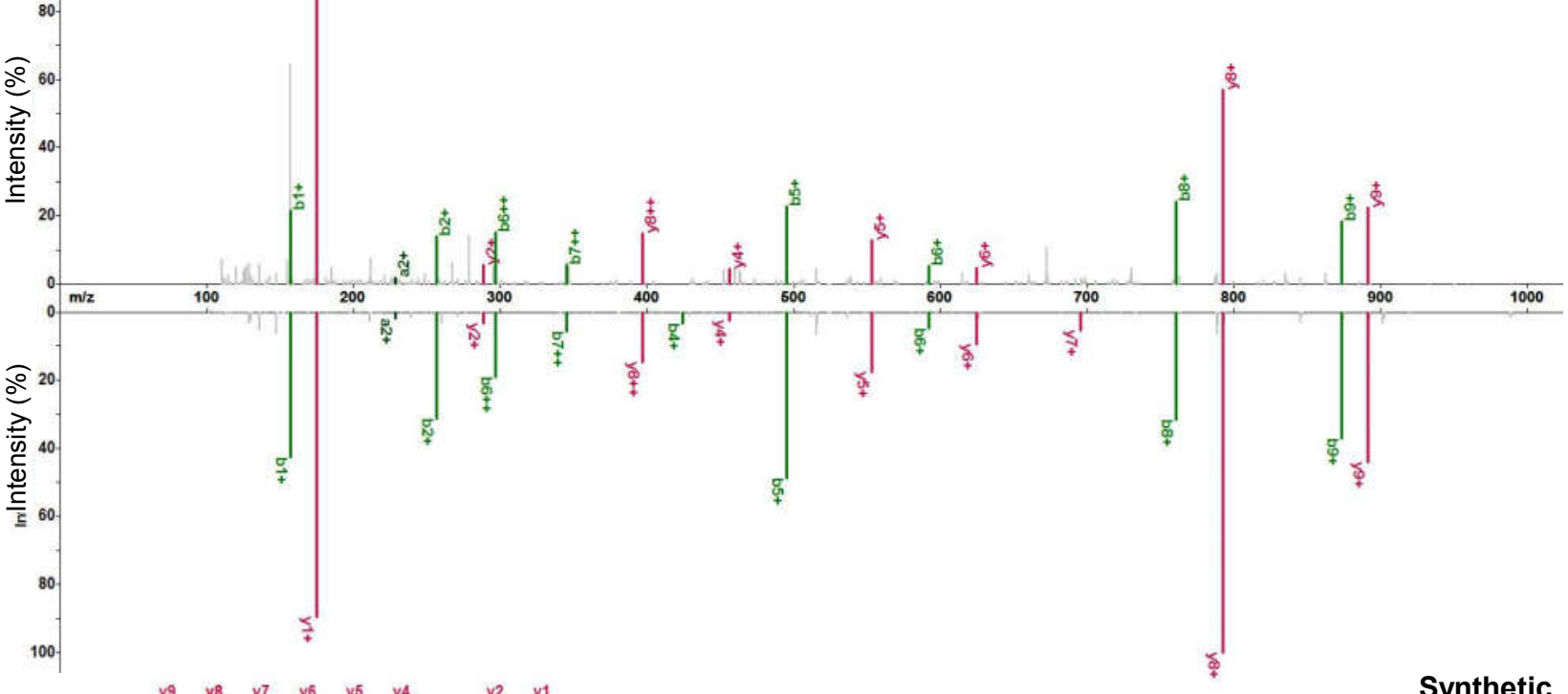

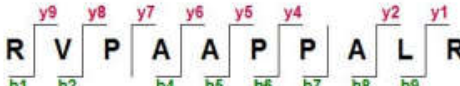

Synthetic

.76E3 3

Precursor m/z: $\mathbf{5 2 4 . 3 2 5}$ Mass error: $-0.011 \mathrm{ppm}$

B 1.34E6 2+

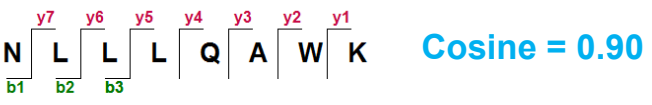

Original

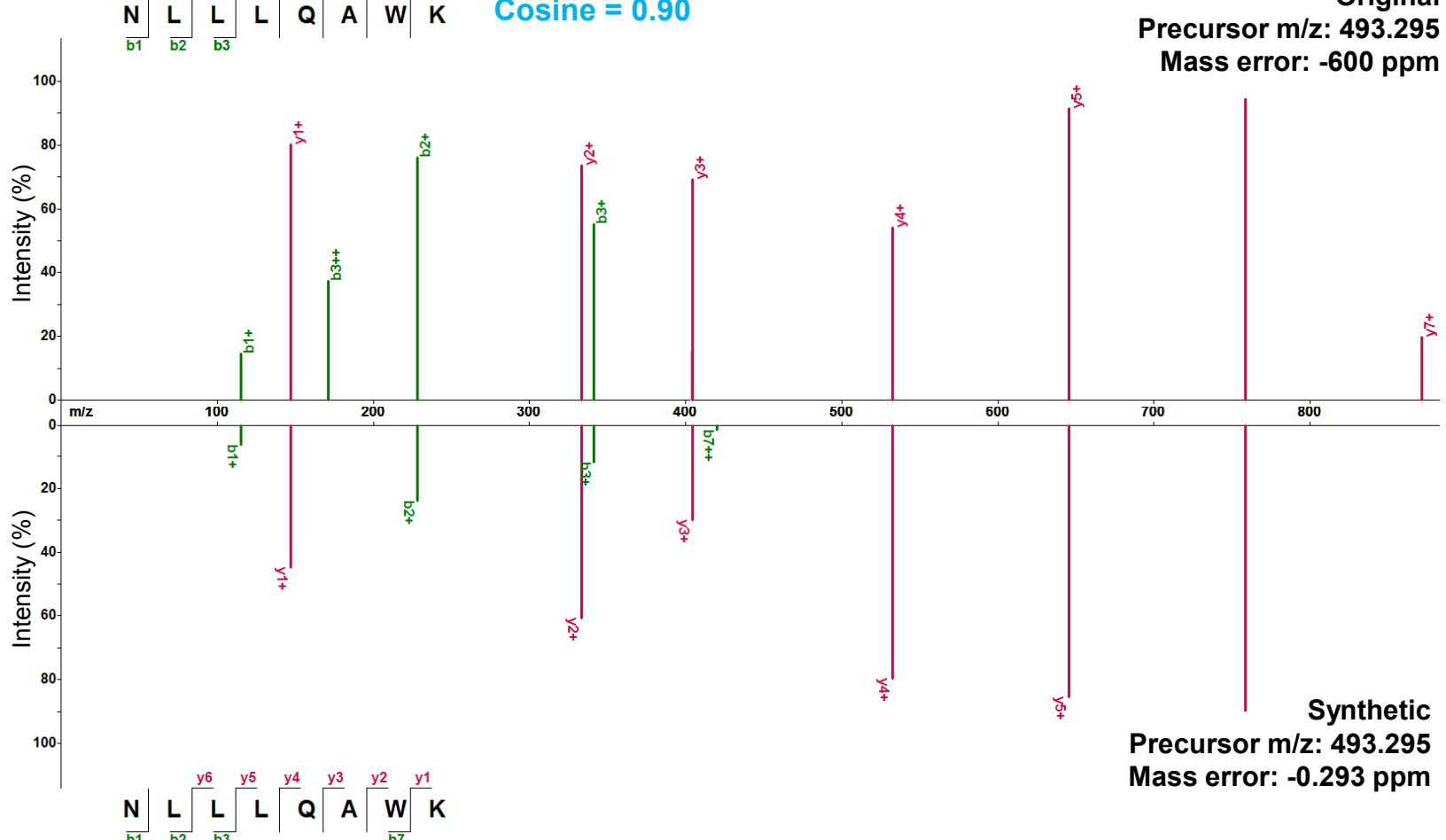

3.96E7 2+ 